\title{
Dual-Threshold Pass-Transistor Logic Design
}

\author{
Lara D. Oliver, Krishnendu Chakrabarty, and Hisham Z. Massoud \\ Duke University Department of Electrical and Computer Engineering \\ Durham, NC 27708, USA \\ $\{$ ldo,krish,massoud\}@ee.duke.edu
}

\begin{abstract}
This paper introduces pass-transistor logic design with dualthreshold voltages. A set of single-rail, fully restored, passtransistor gates are presented. Logic transistors are implemented with low threshold voltages and signal restoration transistors with high threshold voltages. Simulation is used to characterize the leakage power consumption, switching energy, and propagation delay of the proposed gates. A method to reduce circuit power by selectively replacing CMOS gates with the proposed gates is discussed and applied to the ISCAS' 85 benchmark circuits. Relative to circuits composed entirely of conventional CMOS gates, use of the proposed SDPL gates achieves up to $49 \%$ reduction in leakage power and up to $63 \%$ reduction in total power consumption.
\end{abstract}

\section{Categories and Subject Descriptors}

B.6.1 [Logic Design]: Design Styles_Combinational Logic; B.6.3 [Logic Design]: Design Aids-Optimization

\section{General Terms}

Design

\section{INTRODUCTION}

The 2007 International Technology Roadmap for Semiconductors (ITRS) asserts that power consumption is now the major technical problem facing the semiconductor industry [1]. In recent years, the power problem has emerged as one of the fundamental limits facing the future of CMOS integrated circuit design. The aggressive scaling of device dimensions to achieve greater transistor density and circuit speed results in substantial subthreshold and gate oxide tunneling leakage currents. These leakage currents contribute a significant fraction of the total power consumption of CMOS circuits.

Dual-threshold-voltage (dual- $V_{t}$ ) design is a well-known and commonly-utilized leakage reduction strategy in highperformance circuits [2]. The dual- $V_{t}$ design methodology employs low-threshold-voltage $\left(V_{t, L}\right)$ transistors to achieve high speed on critical paths and high-threshold-voltage $\left(V_{t, H}\right)$ transistors to manage leakage elsewhere.

Pass-transistor logic has been considered as a low-power alternative to CMOS logic [3-7]. Pass-transistor design was

\footnotetext{
${ }^{*}$ The work of Lara Oliver was supported in part by a Semiconductor Research Corporation Graduate Fellowship

Permission to make digital or hard copies of all or part of this work for personal or classroom use is granted without fee provided that copies are not made or distributed for profit or commercial advantage and that copies bear this notice and the full citation on the first page. To copy otherwise, to republish, to post on servers or to redistribute to lists, requires prior specific permission and/or a fee.

GLSVLSI'09, May 10-12, 2009, Boston, Massachusetts, USA.

Copyright 2009 ACM 978-1-60558-522-2/09/05 ...\$5.00.
}

found to be well-suited to circuits that contain large proportions of XOR gates and multiplexers, such as arithmetic units, because pass-transistor-based implementations of these functions are more efficient than conventional CMOS implementations [4,7]. Pass-transistor implementations of monotonic logic gates such as NANDs and NORs, however, were found to be slower and consume more power than CMOS implementations $[3,5]$. The leakage of pass-transistor logic implementations of monotonic gates was shown to be much higher than that of CMOS implementations in [6].

In this paper, we develop single-rail, dual- $V_{t}$ passtransistor logic (SDPL) gates and show that they can significantly reduce both dynamic and leakage power consumption relative to conventional and dual- $V_{t}$ CMOS. The proposed SDPL gates are detailed in Section 2. Section 3 introduces the method used to size the transistors of the SDPL gates. An algorithm to substitute SDPL gates for CMOS in order to reduce circuit power consumption is explained in Section 4. Results and discussion are presented in Section 5 and conclusions are drawn in Section 6.

\section{SDPL GATES}

Pass-transistor-based logic gates traditionally contain three components [3-8]. The first is the logic circuitry that implements the functional behavior of the gate. The use of pass-transistors generally enables the implementation of a function with fewer transistors than conventional CMOS logic. The tradeoff associated with the reduced transistor count is that the output range of a pass-transistor is $V_{d d^{-}}$ $V_{t}$, rather than a full rail-to-rail output signal swing. The weak outputs produced by this threshold voltage drop induce significant short circuit currents in subsequent stages and greater noise susceptibility. These effects necessitate the second component of pass-transistor logic: the signal restoration circuitry that restores weak outputs from the logic stage to full-rail voltages. The third component is an output buffer designed to improve drive capability.

As discussed in [6], the signal restoration circuitry and output buffer contribute significantly to the leakage power consumption of a gate. In order to reduce the leakage of SDPL gates, we implement the signal restoration circuitry using low-leakage $V_{t, H}$ transistors and eliminate the output buffer.

The signal restoration circuitry is secondary to the critical path of the gate because the logic circuitry drives the output signal past $50 \%$ of its final value and therefore determines the propagation delay. Consequently, the use of slow $V_{t, H}$ transistors in the signal restoration circuitry does not significantly impact the delay of the entire gate.

The output buffer lies on the critical path and adds a level of inversion to each gate. As a result, the output buffer simultaneously increases propagation delay and consumes additional leakage and dynamic power. To address these issues, the output buffers are omitted from the individual gates. Instead, we place a restriction on SDPL gate use: an SDPL gate is prohibited from driving a signal that is connected to the source of a transistor in another SDPL 


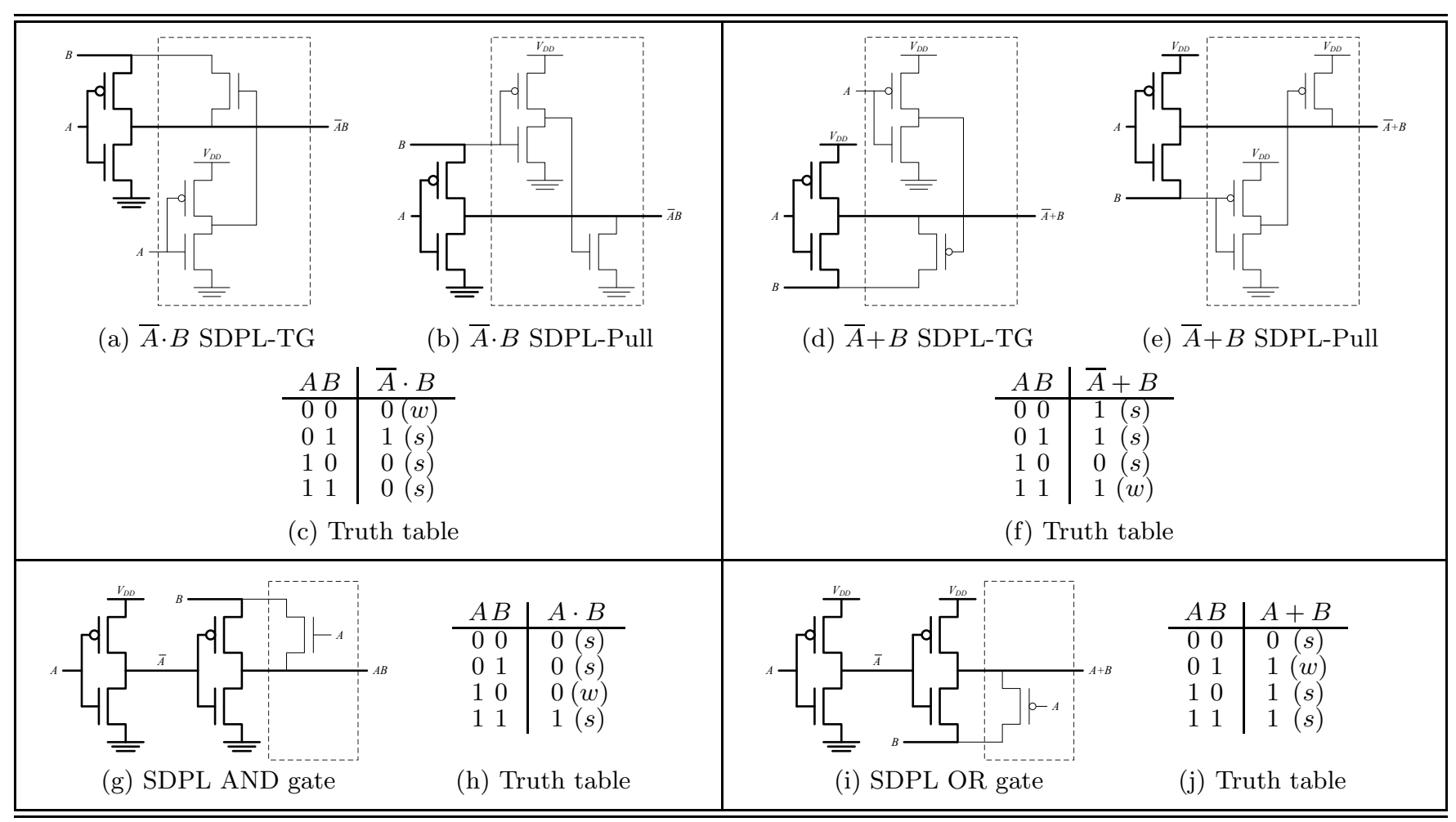

Figure 1: 2-input SDPL gates.

gate. Equivalently, each source-connected SDPL input is required to be driven by a standard CMOS gate.

It should be noted that traditional pass-transistor logic styles restore the output signal using a pull-up or pull-down transistor that is gated by feedback from the output buffer. The SDPL signal restoration transistors are controlled by the gate inputs, rather than the output. Removing the feedback loop eliminates competition between the logic and signal restoration circuitry, simultaneously improving speed and eliminating the need to carefully size the transistors in order to ensure correct functionality. The speed is further increased by the earlier signal arrival time at the gates of the signal restoration transistors.

The remainder of this section details the SDPL gates used in this work. The logic circuitry of each gate contains both a PMOS network and an NMOS network. The $V_{t, H}$ signal restoration circuitry of each gate fully restores the output to achieve full rail-to-rail signal swing.

Transistor-level schematics of two SDPL implementations of the function $\bar{A} \cdot B$ are presented in Figure $1(\mathrm{a})$ and Figure 1(b). Thick lines indicate the $V_{t, L}$ transistors of the logic circuitry and thin lines indicate the $V_{t, H}$ transistors of the signal restoration circuitry. The two implementations have identical logic circuitry and different signal restoration.

The truth table of the logic circuitry is shown in Figure 1(c). Truth table outputs are annotated to indicate whether the output signal of the logic stage is strong, $(s)$, or weak, $(w)$, in the absence of signal restoration. In this discussion, signal strength or weakness refers only to the voltage value achieved in steady state. It does not address the rise or fall time, propagation delay, or drive strength. A strong signal is one that reaches a rail voltage, GND or $V_{d d}$, in steady state. A weak signal is one that is degraded by a threshold voltage drop. Despite this threshold voltage degradation, the logic circuitry outputs a voltage greater than $V_{d d} / 2$ when the logical output is 1 and less than $V_{d d} / 2$ when the output is 0 . The signal restoration circuitry is only necessary to pull the degraded signal to a full-rail value. Therefore, as previously mentioned, the signal restoration circuitry is secondary to the critical path of the gate, enabling it to be implemented with $V_{t, H}$ transistors.

The $\bar{A} \cdot B$ logic circuitry outputs a weak 0 when both inputs $A$ and $B$ are 0 . Thus, an NMOS transistor is required to pull the output down to GND. Figure 1(a) depicts one implementation in which the NMOS transistor is configured as a dual- $V_{t}$ transmission gate, gated by $\bar{A}$, to pass the full $B=0$ signal. The other implementation, shown in Figure 1(b), uses the NMOS transistor as a pull-down transistor, controlled by the $\bar{B}$ signal, to pull the output to GND when $B=0$. The dual- $V_{t}$ transmission gate implementation is denoted SDPL-TG and the pull-down implementation, SDPL-Pull.

Relative to CMOS, the SDPL implementations of the function $\bar{A} \cdot B$ require one less transistor. The pass-transistor implementation requires five transistors: two logic and three signal restoration. The CMOS implementation requires an inverter and a NOR gate, totaling six transistors.

The schematics and truth table of the two SDPL implementations of the function $\bar{A}+B$ are shown in Figures $1(\mathrm{~d})$ 1(f). As indicated in the truth table, the logic circuitry outputs a weak $\bar{A}+B=1$ when inputs $A B=11$. The signal restoration circuitry thus requires a PMOS transistor to pull the output up to $V_{d d}$. The SDPL-TG implementation, shown in Figure 1(d), uses the PMOS transistor to complete the transmisson gate, enabling it to pass full railto-rail $B$ signals. The SDPL-Pull implementation, shown in Figure 1(e), uses the PMOS transistor as a pull-up transistor, controlled by the $\bar{B}$ signal, to pull the output to $V_{d d}$ when $B=1$.

Fully-restored, five-transistor SDPL AND and SDPL OR gates can be derived from the SDPL-TG gates discussed 


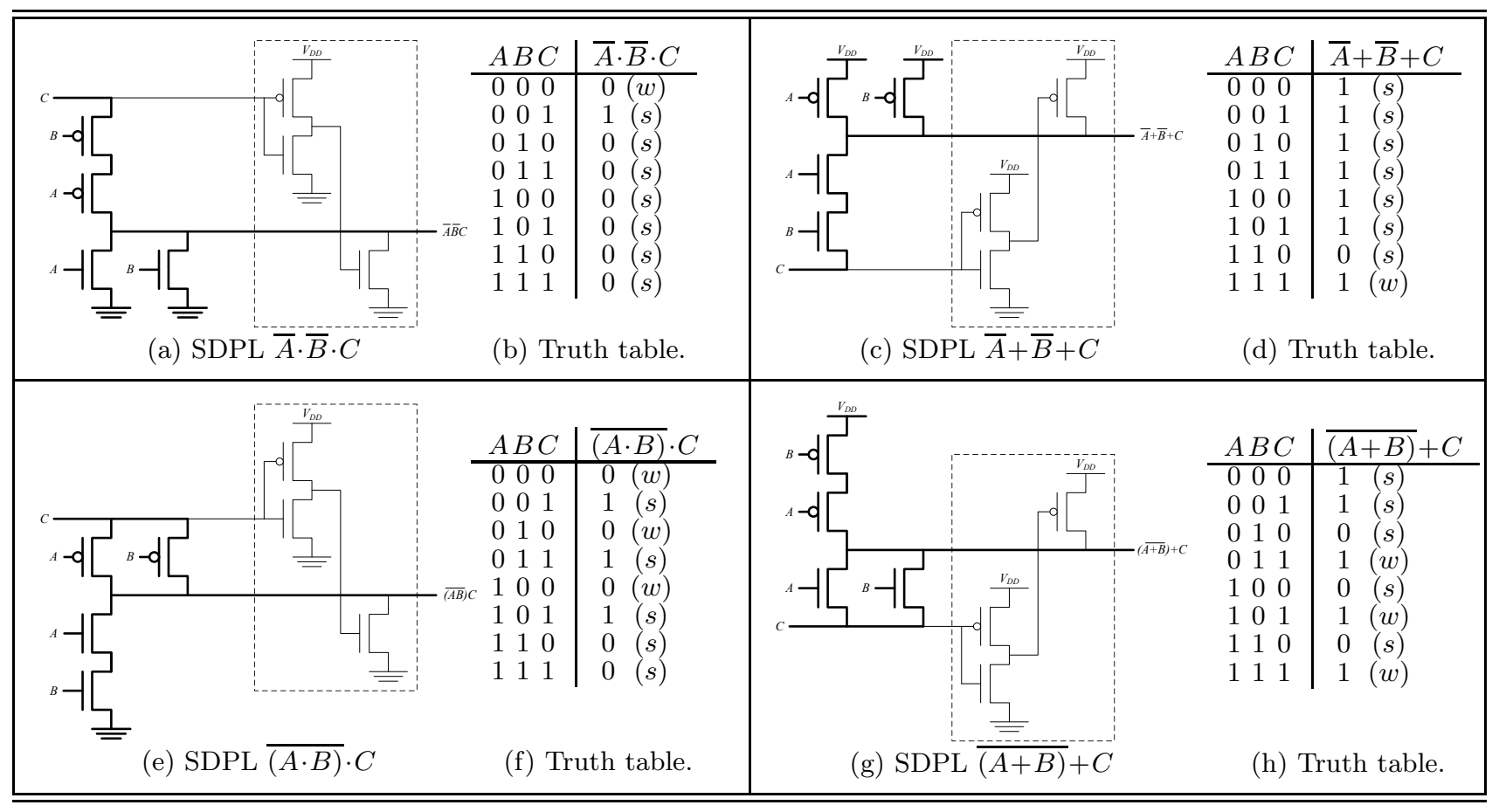

Figure 2: 3-input SDPL gates.

above. Consider the SDPL-TG implementation of $\bar{A} \cdot B$. The signal restoration circuitry inverts the $A$ input to generate the control signal for the $V_{t, H}$ half of the transmission gate. By moving this inverter to drive the logic circuitry and then using the original $A$ input signal to control the signal restoration transistor, we construct a fully-restored, five-transistor AND gate. The same method can be used to construct a five-transistor OR gate.

The schematic and truth table of the SDPL AND and SDPL OR gates are shown in Figures $1(\mathrm{~g})-1(\mathrm{j})$. Unlike the SDPL $\bar{A} \cdot B$ and $\bar{A}+B$ gates, the SDPL AND and OR logic circuitry consists of four $V_{t, L}$ transistors and two delay stages.

The minimum-size layout for each of the 2-input SDPL gates contains three transistors on one diffusion strip and two on the other. The design rules for the $65 \mathrm{~nm}$ dual- $V_{t}$ industry process used in this work permit adjacent $V_{t, L}$ and $V_{t, H}$ transistors on the same diffusion strip. Therefore, the layout area of each 2-input SDPL gate is identical to that of the CMOS gate implementing the same function.

Serial and parallel transistors can be added to the SDPL $\bar{A} \cdot B$ and $\bar{A}+B$ gates to implement 3 -input functions. Figure 2(a) and Figure 2(c) present the schematics of the $\bar{A} \cdot \bar{B} \cdot C$ and $\bar{A}+\bar{B}+C$ gates, constructed by extending the passtransistor stack and adding a parallel rail-connected transistor. As shown in the corresponding truth tables, each gate has one weak state, when both stacked transistors are on and the $C$ input is equal to 0 for the PMOS stack or 1 for the NMOS stack. The output of $\bar{A} \cdot \bar{B} \cdot C$ is always 0 when $C=0$. Therefore, the weak $A B C=000$ input can be restored without introducing any signal conflicts by inverting the $C$ input and using it to control a pull-down transistor. Similarly, the $C$ input can be inverted and used to control a pull-up transistor to restore the weak $A B C=111$ input to the $\bar{A}+\bar{B}+C$ gate.

The schematics of the $\overline{(A \cdot B)} \cdot C$ and $\overline{(A+B)}+C$ gates are shown in Figure 2(e) and Figure 2(g). These gates are implemented by adding a parallel pass-transistor and stacked rail-connected transistor to the 2 -input $\bar{A} \cdot B$ and $\bar{A}+B$ gates. The parallel pass-transistors result in 3 weak states per gate, which occur when either pass transistor is on and attempting to pass a weak signal. However, the $\overline{(A \cdot B)} \cdot C$ gate is like $\bar{A} \cdot \bar{B} \cdot C$ in that all weak outputs occur when $C=0$ and the output is always 0 when $C=0$. Therefore, the same signal restoration circuitry can be used effectively. Similarly, the signal restoration circuitry of $\bar{A}+\bar{B}+C$ can be used for $\overline{(A+B)}+C$.

Like the 2-input SDPL gates, the 3-input gates require one less transistor than the corresponding CMOS implementation of the same function. The layout of a 3-input SDPL gate also requires the same area as the layout of the corresponding CMOS gate.

\section{TRANSISTOR SIZE OPTIMIZATION}

In order to acheive the best performance from the SDPL gates, the transistor widths must be optimized. The sizing optimization problem has two conflicting objectives: power consumption and delay. One method to approach such a problem is to design a weighting function to aggregate the objectives into a single goal. Alternatively, one can search for a set of optimal solutions. Such a set is often defined in terms of Pareto optimality. A solution is Pareto optimal if the objective vector cannot be improved in any dimension without sacrificing another dimension. In this problem, the set of Pareto-optimal solutions are those for which the power cannot be reduced without lengthening delay and the delay cannot be shortened without increasing power. Thus, the Pareto-optimal set is the minimal power-delay curve achievable by the gate.

The Strength Pareto Evolutionary Algorithm (SPEA) utilizes the search methodology of an EA to find the Paretooptimal frontier for an optimization problem with multiple objectives [9]. An enhancement to the algorithm, referred to as SPEA2, improves the fitness function and contains a 
method to retain boundary solutions in the population [10]. SPEA2 has been shown to effectively approximate the Paretooptimal frontier and is used here to search for transistor sizes that optimize power and delay.

HSPICE simulation was used to measure the leakage power, dynamic power, and delay characteristics of each pass-transistor logic style. Devices are modeled with BSIM4, a predictive MOSFET model designed for sub-100nm transistors, using the model parameters from a $65 \mathrm{~nm}$ dual- $V_{t}$ industry process. In order to correctly characterize the realistic behavior of the gate, the input and output pins are driven and loaded, respectively, by inverters. The delay of each input is the maximum propagation delay from that input to the gate ouput. The power of a gate $g$ with $n$ inputs is computed as:

$$
P_{g}=V_{d d} I_{d d}+\sum_{i=1}^{n} V_{\text {in }, i} I_{\text {in }, i}-V_{\text {out }} I_{\text {out }}
$$

Leakage power is then the weighted average of the static power over all input vectors. Dynamic power is determined by measuring the instantaneous power through each transition and computing the switching energy:

$$
E_{s w}=\int_{t_{\text {start }}}^{t_{\text {end }}} P_{g}(t) d t
$$

where $t_{\text {start }}$ is the beginning of the input signal transition and $t_{\text {end }}$ is the end of the output signal transition. The switching energy was measured for all $2^{n} \cdot \sum_{i=1}^{n}\left(\begin{array}{c}n \\ i\end{array}\right)$ singleand multiple-input transitions and averaged. The dynamic power can then be computed as:

$$
P_{d y n}=\alpha f_{c l k} E_{s w}
$$

where $\alpha$ is switching activity and $f_{c l k}$ is clock frequency.

Each SDPL and CMOS gate is optimized using the procedure detailed in this section. The results are presented and discussed in Section 5 .

\section{SDPL-SUBSTITUTION ALGORITHM}

The objective of SDPL-Substitution is to reduce the power consumption of a large combinational circuit without altering the logical functionality or increasing the longest path delay. The optimal solution to this problem would be the circuit configuration that results in the minimum total power consumption. However, the SDPL-Substitution problem can be shown to be NP-complete through polynomial transformation to the multiple choice knapsack problem. (The proof is omitted here due to lack of space.) Therefore, a greedy heuristic algorithm is used to replace CMOS gates with SDPL to reduce the total power of a circuit.

The heuristic algorithm begins by computing the priority of each edge as the product of the edge slack and the forward level of its end node. Because a large slack value tolerates delay increases and the algorithm traverses backward through the circuit, this priority metric allows the algorithm to select a starting point with high likelihood of successful substition. The algorithm then attempts to substitute an SDPL gate for the node at the end of the highest priority edge, $e_{p, \max }$.

A combination of De Morgan's laws and bubble pushing are used to substitute each of the library gates for the initial gate while maintaining the logical equivalence of the circuit. The library gate is substituted for the existing gate and then bubbles (inversions) are placed on input and output edges as necessary to ensure that the resulting circuit implements the same function as the initial circuit.
A substitution is classified as valid if it satisfies four criteria. First, the substitution does not cause any edge to have a negative slack value. Second, the substitution does not result in an SDPL gate driving the source-connected input of a subsequent gate. Third, the substitution reduces the total circuit power relative to the initial state. Fourth, the substitution only generates bubbles that can be pushed backward through the circuit toward the primary inputs. For example, substituting an AND gate for a NAND gate would be invalid because it would require a bubble to be placed on the output edge. The last requirement is necessary in order to limit the algorithm search space and execution time.

After all library gates have been tried, the valid substitutions are ranked in order of their power reduction relative to the initial state. The initial gate is then replaced with the valid substitution that results in the greatest power savings. Any bubble that is placed on an input edge is then pushed back to invert the ouput of the driving gate. If the driver is an inverter, it is removed from the circuit. Otherwise, the algorithm attempts to substitute each library gate for the modified driver, as described in the previous paragraphs. If no valid substitutions are found, the algorithm returns to the load gate and tries to justify the valid substitution with the next-highest power savings.

This process continues until a success or failure terminating condition is reached. A set of substitutions is successful when all bubbles have been justified, rendering further bubble pushing unnecessary. A substitution attempt fails when no further valid substitutions remain. To prevent exponential complexity, the number of times any gate can changed while attempting to justify a given start point, $e_{p, \max }$, is limited to a constant. If this number of substitutions is reached, the substitution attempt fails.

On successful termination, the circuit changes are committed and all of the edges traversed by the pushed bubbles are marked. When the substitution attempt fails, the circuit is reverted to its previous state and the high-priority edge that initiated the substitution attempt is marked. Marking the successful and failed edges prevents the algorithm from repeating efforts in subsequent iterations.

It should be noted that when a bubble is pushed back to a gate with a fanout greater than one, the bubble must also be pushed sideways to the input nodes of the other loads driven by the gate. In these cases, substitution is attempted for each of the other loads with the additional restriction that a bubble may not be pushed back to the input edge from which it came.

The substitution procedure is repeated until no unmarked edges remain. Assuming that at least one set of substitutions is successful, the resulting circuit contains a combination of CMOS and SDPL gates, and, in comparison to the original circuit, is logically equivalent, consumes less power, and has the same or shorter maximum delay.

For a given start point, the algorithm could attempt up to $C$ substitutions for each node (vertex), $V$, in the circuit. Consequently, an iteration is linear in $V$. The number of start points is linear in the number of edges, $E$, in the circuit graph, making the overall algorithm complexity $O(V \cdot E)$.

\section{SIMULATION RESULTS}

Figure 3 presents the Pareto-optimal $P_{\text {tot }}$-delay curves of the size-optimized SDPL-TG and CMOS 2-input gates. The SDPL-Pull implementations had slightly higher power and delay than the corresponding SDPL-TG implementations and are thus omitted from the figure for clarity. For a given function, the power-delay curves of the SDPL and CMOS implementations are shown with the same marker. 


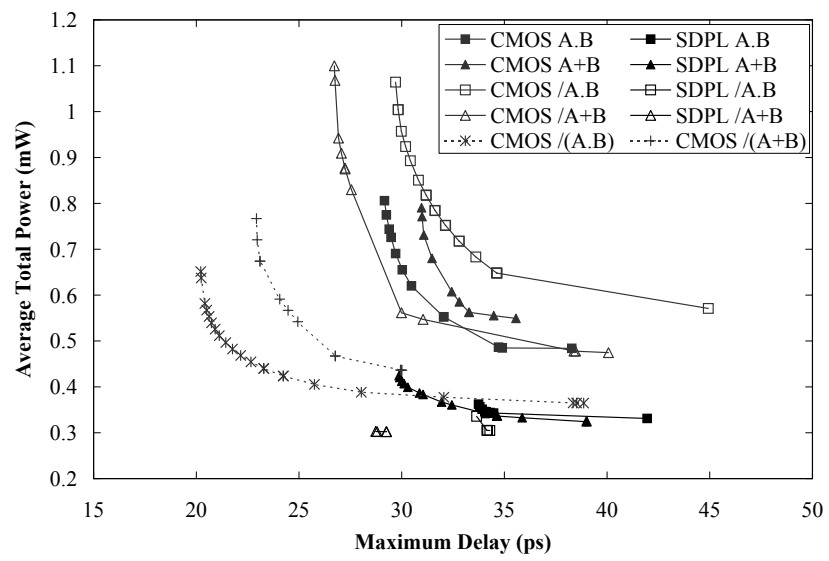

Figure 3: $P_{t o t}$-delay curves of optimized 2-input SDPL-TG and CMOS gates.

The black curve indicates the SDPL implementation and the CMOS curve is grey. The power-delay curves of CMOS NAND and NOR gates are also shown for reference.

The minimum delay of an SDPL gate is limited by the size of the gate that drives the source-connected input. All drivers in these simulations were of minimum size. Consequently, the threshold after which an increase in transistor size will increase power consumption without a corresponding decrease in delay is much lower for the SDPL gates than the CMOS ones. This effect is manifested in two ways. First, the SDPL power-delay curves are much flatter than those of the CMOS gates. Second, the CMOS implementations achieve the shortest delay for all functions except $\bar{A}+B$.

The SDPL implementation of each 2-input function consumes significantly less average total power than the corresponding CMOS implementation. This difference in power is present at every delay achievable by the SDPL implementation of each function. The minimum power consumption of each SDPL 2-input gate is also less than that of the CMOS NAND and NOR gates.

For a given gate, each of the size-optimized implementations that compose the power-delay curve shown in Figure 3 has a different power-delay product (PDP). The sized implementations of each gate that have the best PDP are compared in Table 1. The leakage power, switching energy, and total power of the SDPL implementation is less than that of the CMOS implementation for all functions under consideration. The PDP of each SDPL gate is less than that of the corresponding CMOS gate, as well.

Among the 2-input gates, the maximum difference in total power occurs between the SDPL-TG and CMOS implementations of $\bar{A} \cdot B$ and is $53 \%$. The minimum total power difference was $29 \%$, for $A \cdot B$. The difference in leakage power ranges from $10 \%$ for $A \cdot B$ to $51 \%$ for $A+B$. For both $\bar{A} \cdot B$ and $\bar{A}+B$, the SDPL-Pull implementation consumes $4 \%$ more power than the SDPL-TG implementation. The delay of each SDPL 2-input gate is comparable to that of the CMOS implementation of the same function. The SDPL implementation is slightly faster than CMOS for all cases except $A+B$ and the SDPL-Pull implementation of $\bar{A} \cdot B$.

Among the 3 -input gates, the gates with parallel pass transistors have the greatest difference between SDPL and CMOS total power. The difference in total power ranges from $35 \%$ for $\bar{A}+\bar{B}+C$ to $65 \%$ for $\overline{(A+B)}+C$. The difference in leakage power ranges from $15 \%$ for $\overline{(A+B)}+C$ to $47 \%$ for $\bar{A} \cdot \bar{B} \cdot C$. Both SDPL gates with parallel pass transistors also improve delay relative to the CMOS implementations.
For the SDPL implementations that contain pass transistor stacks, the gate with an NMOS stack, $\bar{A}+\bar{B}+C$, has comparable delay to its CMOS counterpart, while the gate with a PMOS stack, $\bar{A} \cdot \bar{B} \cdot C$, exhibits longer delay.

A number of factors contribute to the difference in power consumption between the SDPL and CMOS implementations. The SDPL leakage is less because fewer transistors are required to implement a given function and $20-60 \%$ of the SDPL transistors are lower-leakage $V_{t, H}$ transistors. Furthermore, for $\bar{A} \cdot B$ and $\bar{A}+B$, there is negligble subthreshold leakage through the $V_{t, L}$ logic transistors when the voltage of the input connected to the source of the logic passtransistors is equal to the rail voltage connected to the source of the other logic transistor. This occurs for two of the four possible input vectors: when $B=0$ for $\bar{A} \cdot B$ and when $B=1$ for $\bar{A}+B$. This also occurs for half of the input vectors of four 3-input gates: when $C=0$ for $\bar{A} \cdot \bar{B} \cdot C$ and $\overline{(A \cdot B)} \cdot C$ and when $C=1$ for $\bar{A}+\bar{B}+C$ and $\overline{(A+B)}+C$. In addition, there is negligble gate-oxide tunneling leakage in the $V_{t, L}$ logic transistors when all input voltages and the output voltage are equal because there is no voltage drop between the gate and source or drain of either transistor. This occurs when $A B=00$ for $\bar{A} \cdot B, A B=11$ for $\bar{A}+B$, when $A B C=000$ for $\bar{A} \cdot \bar{B} \cdot C$ and $\overline{(A \cdot B)} \cdot C$, and when $A B C=111$ for $\bar{A}+\bar{B}+C$ and $\overline{(A+B)}+C$.

The SDPL switching energy is less than that of CMOS primarily due to the differing inverters contained in each gate. The inverters in the SDPL gates are smaller and have smaller load capacitances, thereby consuming less short circuit and capacitive charging power. In addition, very little power is dissipated within the gate due to the switching of the source-connected input.

The power savings demonstrated by the gate-level comparison also occurs when SDPL gates are substituted for CMOS gates in large circuits. Figure 4 presents the total and leakage power savings resulting from the application of the SDPL-Substitution algorithm to the ISCAS'85 benchmarks. The power savings are expressed as a percent-

Table 1: Power and delay comparison of minimumPDP CMOS and SDPL gates.

\begin{tabular}{|r|r|rrrr|}
\hline \hline $\begin{array}{r}\text { Logic } \\
\text { Function }\end{array}$ & Gate Type & $\begin{array}{r}\text { Delay } \\
(\mathrm{ps})\end{array}$ & $\begin{array}{r}P_{\text {leak }} \\
(\mathrm{nW})\end{array}$ & $\begin{array}{r}E_{\text {sw }} \\
(\mathrm{fJ})\end{array}$ & $\begin{array}{r}P_{\text {tot }} \\
(\mathrm{nW})\end{array}$ \\
\hline \multirow{A}{A}{$\cdot B$} & CMOS & 34.6 & 9.56 & 1.60 & 648.1 \\
& SDPL-Pull & 37.0 & 7.01 & 0.81 & 332.1 \\
& SDPL-TG & 34.2 & 5.99 & 0.75 & 305.2 \\
\hline \multirow{2}{*}{$+B$} & CMOS & 30.0 & 11.33 & 1.38 & 561.6 \\
& SDPL-Pull & 28.4 & 6.55 & 0.80 & 324.6 \\
& SDPL-TG & 28.7 & 6.19 & 0.74 & 302.8 \\
\hline \multirow{2}{*}{$A \cdot B$} & CMOS & 34.7 & 6.63 & 1.20 & 486.6 \\
& SDPL & 34.1 & 5.97 & 0.85 & 345.8 \\
\hline \multirow{2}{*}{$A+B$} & CMOS & 33.3 & 9.14 & 1.38 & 562.6 \\
& SDPL & 34.6 & 4.47 & 0.83 & 336.6 \\
\hline $\bar{A} \cdot \bar{B} \cdot C$ & CMOS & 46.4 & 15.30 & 1.70 & 694.3 \\
& SDPL & 59.5 & 8.16 & 0.69 & 285.8 \\
\hline $\bar{A}+\bar{B}+C$ & CMOS & 40.5 & 9.17 & 1.00 & 409.4 \\
& SDPL & 40.4 & 7.36 & 0.64 & 264.2 \\
\hline$\overline{(A \cdot B)} \cdot C$ & CMOS & 50.4 & 14.33 & 2.06 & 839.8 \\
& SDPL & 44.4 & 9.34 & 0.81 & 331.6 \\
\hline$\overline{(A+B)}+C$ & CMOS & 46.2 & 14.29 & 2.10 & 853.0 \\
& SDPL & 36.9 & 12.01 & 0.72 & 299.8 \\
\hline \hline
\end{tabular}



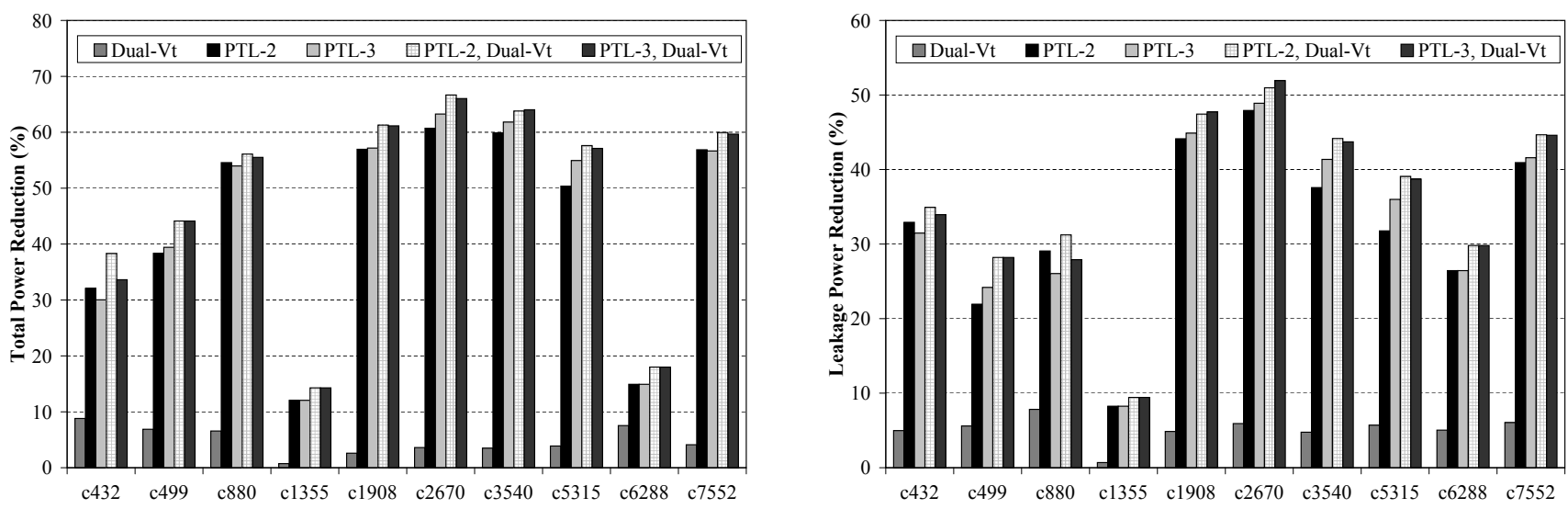

Figure 4: Percentage reduction in total and leakage power achieved through SDPL substitution.

age reduction relative to the all- $V_{t, L}$ CMOS circuit implementation. For comparison, the Iterative Max-Cut dual- $V_{t}$ algorithm detailed in [11] was also applied to the benchmarks. The power savings achieved by that algorithm are presented as the "Dual- $V_{t}$ " results. The results of the SDPLSubstitution algorithm using only 2 -input SDPL gates and using all 2- and 3-input SDPL gates are presented as "PTL2" and "PTL-3", respectively. Finally, the Iterative Max-Cut algorithm was applied to the CMOS gates remaining in the PTL-2 and PTL-3 results to achieve further power savings. The results of these applications are presented as "PTL-2, Dual- $V_{t}$ " and "PTL-3, Dual- $V_{t}$ ".

To test the accuracy of the power models used by the algorithm, one-thousand random input transitions were simulated in SPICE on each initial and final benchmark circuit. The average leakage estimation error was $1 \%$ with a maximum error of $4 \%$. The average dynamic power estimation error was $3 \%$ with a maximum error of $7 \%$. The delay estimated by the model was worst-case delay and therefore longer than the simulated delay.

The use of SDPL gates saved significantly more power than standard dual- $V_{t}$ for all benchmark circuits. On average, dual- $V_{t}$ saved $5 \%$ leakage power, while the SDPL results saved $33 \%$. The leakage power savings achieved by the dual- $V_{t}$ algorithm is less than that reported in [11] and other works describing dual- $V_{t}$ algorithms because the device-level leakage reduction of the process used in this work is low relative to the processes used in those works. However, the number of transistors assigned high- $V_{t}$ values is comparable to the results presented in [11]. It is therefore reasonable to assume that a process with higher leakage savings would generate similar results to those presented in Figure 4.

The use of SDPL gates alone generated $44 \% P_{\text {tot }}$ savings on average with a maximum savings of $61 \%$ for PTL-2 and $63 \%$ for PTL-3. In some circuits, the 3 -input gates saved additional power relative to 2 -input gates alone. In other circuits, the inclusion of 3-input gates reduced the total power savings. These cases occurred when a large portion of the delay slack on a given path was devoted to a single 3 -input SDPL gate when that same slack could have been divided among multiple 2-input SDPL gates.

The use of SDPL gates in conjunction with dual- $V_{t}$ CMOS gates generated additional power savings between $1.5 \%$ and $7 \%$. The extra power savings generally came from CMOS gates that were unable to be converted to SDPL because they were driving the source-connected input of an SDPL gate, driving a large load, or when it would have been necessary to insert an inverter in order to use an SDPL gate and retain correct logic.

\section{CONCLUSIONS}

This work introduced single-rail dual- $V_{t}$ pass-transistor logic design and demonstrated that it can achieve significant leakage and dynamic power savings relative to conventional and dual- $V_{t}$ CMOS. SDPL logic gates were designed using $V_{t, L}$ transistors to implement the logic circuitry and $V_{t, H}$ transistors for signal restoration. The gates were sized for optimal delay and power consumption and compared to the CMOS implementations of the same functions. An algorithm was developed to substitute SDPL gates for CMOS in order to reduce the total power of a circuit without increasing delay or changing the logical functionality. The algorithm was applied to the ISCAS' 85 benchmark circuits. Results showed that the use of SDPL gates in place of CMOS can achieve up to $49 \%$ leakage power reduction and up to $63 \%$ total power reduction.

\section{REFERENCES}

[1] ITRS (2007). http://www.itrs.net.

[2] T. Karnik et al., "Total power optimization by simultaneous dual- $v_{t}$ allocation and device sizing in high performance microprocessors," in Design Automation Conference, 2002. Proceedings. 39th, New Orleans, LA, USA, 2002, pp. 486-491.

[3] R. Zimmermann and W. Fichtner, "Low-power logic styles: CMOS versus pass-transistor logic," IEEE J. Solid-State Circuits, vol. 32, no. 7, pp. 1079-1090, Jul. 1997.

[4] K. Yano et al., "Top-down pass-transistor logic design," IEEE J. Solid-State Circuits, vol. 31, no. 6, pp. 792-803, Jun. 1996

[5] M. Anis, M. Allam, and M. Elmasry, "Impact of technology scaling on CMOS logic styles," IEEE Trans. Circuits and Systems II: Analog and Digital Signal Processing, vol. 49, no. 8, pp. 577-588, Aug. 2002.

[6] G. Merrett and B. M. Al-Hashimi, "Leakage power analysis and comparison of deep submicron logic gates," in Proc. 14th Int. Workshop on Power, Timing, Modeling, Optimization, and Simulation (PATMOS), Sep. 2004, pp. 198-207.

[7] A. P. Chandrakasan, S. Sheng, and R. W. Brodersen, "Low-power CMOS digital design," IEEE J. Solid-State Circuits, vol. 27, no. 4, pp. 473-484, Apr. 1992.

[8] H. Al-Hertani, D. Al-Khalili, and C. Rozon, "Leakage power dissipation in UDSM logic gates," in Proc. 3rd IASTED Int Conference on Circuits, Signals, and Systems, Oct. 2005.

[9] E. Zitzler and L. Thiele, "Multiobjective evolutionary algorithms: a comparative case study and the strength pareto approach," IEEE Trans. Evolutionary Computation, vol. 3, no. 4, pp. 257-271, Nov 1999.

[10] E. Zitzler, M. Laumanns, and L. Thiele, "Spea2: Improving the strength pareto evolutionary algorithm," in TIK-Report No. 103, Zurich, Switzerland: Swiss Federal Institute of Technology, 2001.

[11] Q. Wang and S. B. K. Vrudhula, "Algorithms for minimizing standby power in deep submicrometer, dual-vt CMOS circuits," TCAD, vol. 21, no. 3, pp. 306-318, March 2002. 